\title{
ClinLabGeneticist: a tool for clinical management of genetic variants from whole exome sequencing in clinical genetic laboratories
}

\author{
Jinlian Wang, Jun Liao, Jinglan Zhang, Wei-Yi Cheng, Jörg Hakenberg, Meng Ma, Bryn D. Webb, \\ Rajasekar Ramasamudram-chakravarthi, Lisa Karger, Lakshmi Mehta, Ruth Kornreich, George A. Diaz, Shuyu Li, \\ Lisa Edelmann ${ }^{*}$ and Rong Chen ${ }^{*}$
}

\begin{abstract}
Routine clinical application of whole exome sequencing remains challenging due to difficulties in variant interpretation, large dataset management, and workflow integration. We describe a tool named ClinLabGeneticist to implement a workflow in clinical laboratories for management of variant assessment in genetic testing and disease diagnosis. We established an extensive variant annotation data source for the identification of pathogenic variants. A dashboard was deployed to aid a multi-step, hierarchical review process leading to final clinical decisions on genetic variant assessment. In addition, a central database was built to archive all of the genetic testing data, notes, and comments throughout the review process, variant validation data by Sanger sequencing as well as the final clinical reports for future reference. The entire workflow including data entry, distribution of work assignments, variant evaluation and review, selection of variants for validation, report generation, and communications between various personnel is integrated into a single data management platform. Three case studies are presented to illustrate the utility of ClinLabGeneticist. ClinLabGeneticist is freely available to academia at http://rongchenlab.org/software/clinlabgeneticist.
\end{abstract}

\section{Background}

Molecular genetic testing is playing an increasingly important role in medicine. Due in large part to the breakthrough of genome and exome sequencing technologies, the scope of clinical genetic testing has been expanded from its traditional niche in rare Mendelian disorders to a broad application in complex disease and personalized medicine $[1,2]$. Currently, clinical genetic testing is utilized for a variety of purposes including follow-up to newborn screening for the identification of genetic disease that may affect a child's long-term health or survival, carrier screening for inherited recessive and $\mathrm{X}$-linked diseases, diagnostic testing for symptomatic individuals, predictive testing of asymptomatic individuals for late-onset and complex diseases, pharmacogenetic testing for drug responses with respect to efficacy or adverse

\footnotetext{
* Correspondence: lisa.edelmann@mssm.edu; rong.chen@mssm.edu Department of Genetics and Genomic Sciences, Icahn Institute for Genomics and Multiscale Biology, Icahn School of Medicine at Mount Sinai, New York, NY, USA
}

effects, and testing of tumor biopsies to determine somatic alterations for cancer classification, prognosis, and development of personalized treatment options [1].

There are a number of challenges in applying whole exome sequencing (WES) in clinical genetic testing. Although most clinical genetic testing laboratories follow the guidelines from national and international agencies such as American College of Medical Genetics (ACMG), College of American Pathologists (CAP), and Clinical and Laboratory Standard Institute (CLSI), tools are lacking to bridge these guidelines and clinical practice. In addition, there are a large number of variants of uncertain significance (VUS). As basic research accelerates with improved technology and more discoveries are made toward the genetic basis of human diseases, it is critical to incorporate the most updated and comprehensive genetic variant findings into clinical genetic testing. In addition, previously completed testing reports may need to be updated when new information becomes available on the function and pathogenicity of the identified 
variants. Genetic testing in clinical laboratories involves a complicated process, which requires efficient data management and process management with seamless coordination and communication between various personnel. A final report for each patient is expected to precisely summarize the current knowledge surrounding the variants and their clinical implication with supporting evidence, and the report should be generated in a comprehensive fashion. Currently, although many clinical laboratories use commercial tools to annotate variants in a semi-automated fashion, variant assessment still involves manual inspection of different online databases and copy-paste of relevant content into the report. Many laboratories still use Excel files to manage variant datasets. As the volume of WES testing increases, this practice is inefficient, error-prone, and unscalable. Therefore, in order to facilitate the implementation of WES-based genetic testing, an integrative tool is essential to provide a comprehensive data source for variant assessment, and to automate, therefore, enhance the efficiency of the process and reduce potential errors that may arise in handling large datasets.

There are several commercial tools currently available for variant analysis and interpretation. For example, Ingenuity Variant Analysis tool by QIAGEN [3], Geneticist Assistant by SoftGenetics [4], VarSeq by Golden Helix [5], VarSim by Bina Technology [6], ANNOVAR Tute annotation from Tute Genomics [7], and The Exchange by NextCode [8] allow users to import VCF files after initial processing of sequencing data, followed by variant filtering based on data-related parameters such as supporting sequence reads or allele frequency. Subsequently, users can further explore the data to examine if the variants are present in various databases such as dbSNP [9] for known polymorphisms and their population allele frequencies, or in disease related variant databases such as ClinVar [10], OMIM [11], HGMD [12], and COSMIC [13]. Potential functional consequences of the variants can also be assessed using methods such as SIFT [14], PolyPhen [15], and SeqHBase [16, 17]. Most of these tools also provide a genomic viewer for visualization of variants and sequence alignment. Some of the tools, such as NextCode's The Exchange, even allow usercontrolled data sharing. However, most of these tools are designed primarily for research purposes and others do not meet all the needs of a clinical laboratory. GeneInsight Suite is a tool developed to support use of DNA-based genetic testing by clinical laboratories and health providers [18]. However, it was primarily designed for clinical variant data storage, variant classification, and report generation.

Previously, we reported a comprehensive validation study for WES implementation in the Genetic Testing Laboratory at Mount Sinai [19]. We tested parameters that measure the reproducibility of the sequencing platform as well as the informatics pipelines. Our evaluation focused on SNV and small indel detection for a single workflow across multiple technical replicates. This study validated the analytic performance of WES according to the recommended guidelines [20], and established the foundation of WES-based genetic testing at Mount Sinai. In this report, we describe a tool named ClinLabGeneticist specifically designed to enable and facilitate WES testing in a clinical genetic laboratory setting. We have established a comprehensive data repository for variant annotation including all of the publicly available databases, to our knowledge, for non-disease or disease-related variants. This application provides a platform to automate data management and process management for the highly complex genetic testing workflow, significantly improving the efficiency of clinical WES testing.

\section{Implementation}

\section{WES and ClinLabGeneticist workflow}

The overall WES workflow at Mount Sinai Genetic Testing Laboratory is illustrated in Fig. 1. For whole exome sequencing, genomic DNA was extracted from the peripheral blood samples of patients and exonic regions were enriched by Agilent SureSelect XT Human All Exon V5 capture library. Massively parallel sequencing was performed on an Illumina HiSeq2000/2500 with a 100 bp paired-end protocol. The genome analysis pipeline or GAP, which is based on the 1000 Genomes data analysis pipeline and is composed from the widely-used open source software projects including bwa, Picard, GATK, snpEff, BEDTools, PLINK/SEQ, and custom-developed software was used for variant calling and annotation [19]. VCF files generated by GAP were then uploaded into Ingenuity Variant Analysis tool (QIAGEN) for further variant filtering. Based on patients' clinical and family history, multiple analyses were performed in Ingenuity including HGMD analysis (for searching disease-causing mutations or DM reported in HGMD database), de novo analysis (for searching de novo variants), dominant analysis (for dominant inheritance pattern), recessive analysis, compound heterozygous analysis (both for recessive inheritance and $\mathrm{X}$-linked patterns), and secondary finding analysis (based on ACMG incidental finding gene list) [21]. Variant lists generated by these Ingenuity analyses were then used as input for the ClinLabGeneticist software. Users can also upload input files directly into ClinLabGeneticist after variant filtering using tools such as Cartagenia Bench Suite [22] or the Clinical Sequence Analyzer (CSA) from NextCode [23]. The input file format (Additional file 1: Table S1) should include the following columns: chromosome number, chromosome coordinate, reference allele, sample allele, gene symbol, transcript ID, nucleotide alteration, amino acid alteration, SIFT functional prediction, 


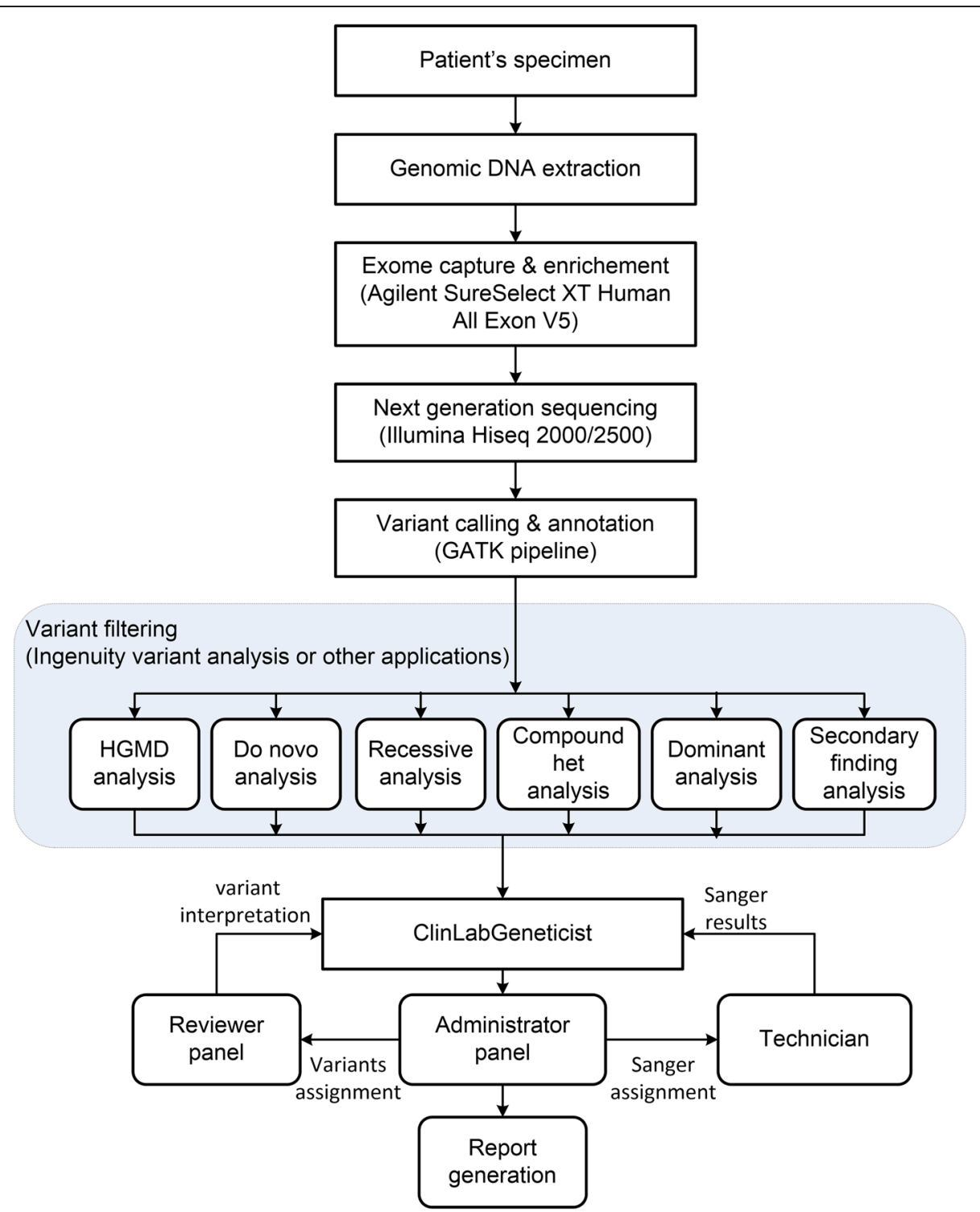

Fig. 1 WES workflow at Mount Sinai Genetic Testing Laboratory

PolyPhen-2 functional prediction, conservation phyloP p-value, dbSNP ID, and 1000 genome allele frequency. ClinLabGeneticist supports analysis of variants generated using various sequencing platforms including Ion Torrent, Agilent, Nimblegen, and others.

The architecture and functionalities of ClinLabGeneticist are depicted in Fig. 2. Two dashboards were designed for the administrators and the reviewers. The dashboard for the administrators enables them to accomplish the following responsibilities: (1) Upload variant data derived from a patient sample; generate a master table with variant annotations automatically retrieved from our annotation repository; select relevant annotation databases for each variant; distribute variants to different groups of reviewers; and notify reviewers of their tasks and deadlines. Each variant can be assigned to at least two reviewers for independent review. (2) Examine the results submitted by the reviewers, merge results, and highlight discordant interpretations on the same variant by different reviewers. (3) Set up reviewer group meetings for discussion, resolve discrepancies in variant interpretations, select variants for validation by Sanger sequencing, and trigger the validation process. (4) Push results to the laboratory director for final decisions on what variants to report and their interpretations, and generate variant tables for final reports.

The reviewers' dashboard is designed to allow reviewers to review the assigned variants, provide variant analysis results and interpretations through the dashboard, and discuss with other reviewer assigned on the same variant. 


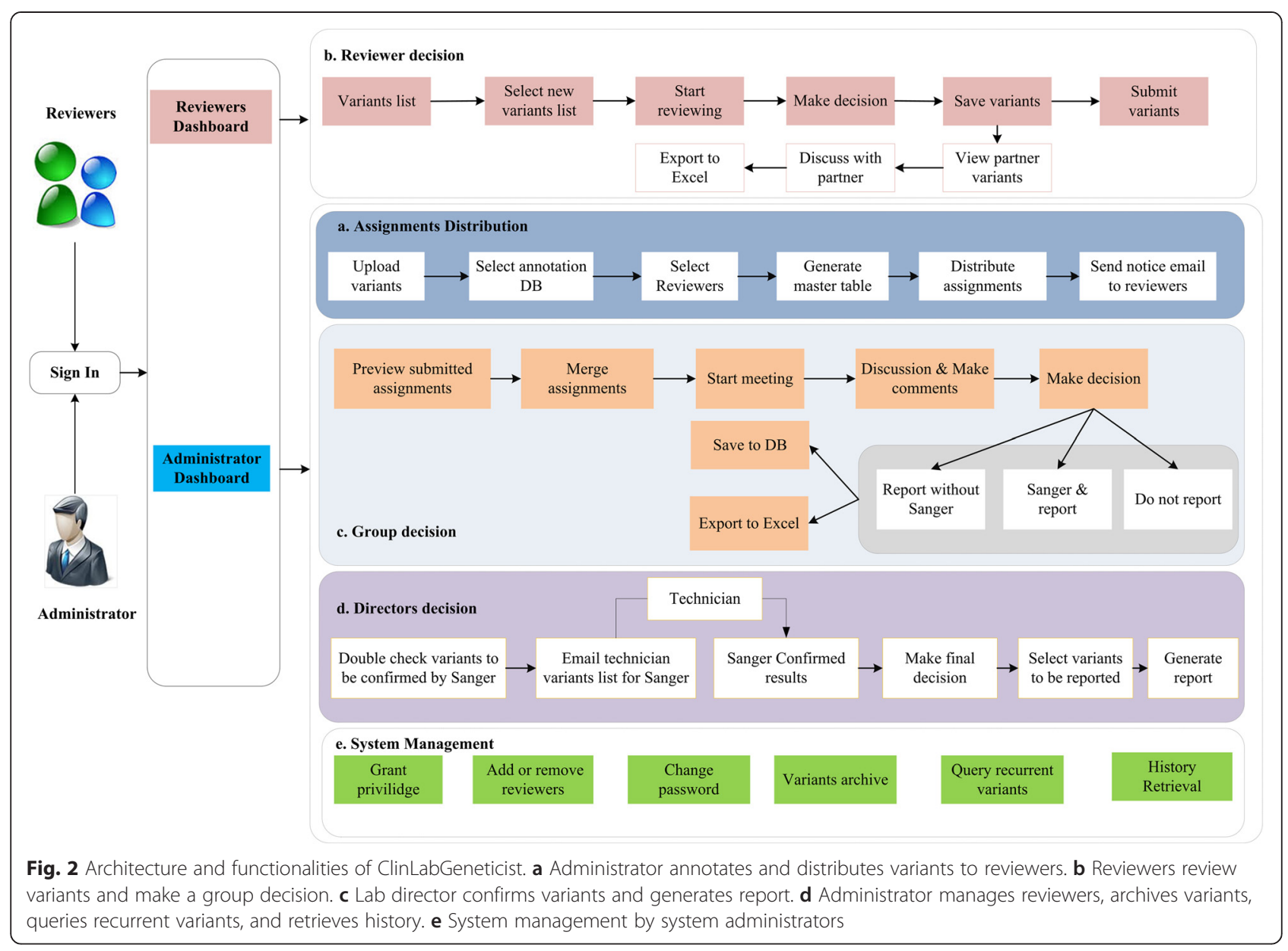

The system is designed to auto-save reviewers' variant annotation every $30 \mathrm{~s}$. The IGV viewer is integrated into ClinLabGeneticist to display sequence alignment for visual inspection of variants. Hyperlinks are set up for variant annotations to their corresponding external databases (for example, dbSNP, OMIM, ClinVar, and so on) upon which the annotation is based. In addition, the chromosome location of the variant is linked to the UCSC browser, gene symbol is linked to the GeneCards website for more detailed gene description, and each gene is linked to NCBI PubMed for relevant literature. Integration of these links and the IGV viewer provides tremendous convenience for the reviewers so they can perform all required tasks within the same software system without having to manually launch different tools separately.

The system is managed by a system administrator whose responsibilities include granting privileges, adding or removing reviewers, and managing variant archives.

\section{Variant annotation resources in ClinLabGeneticist}

We developed a comprehensive variant annotation repository. The included databases, datasets, and annotation features are listed in Table 1 and Additional file 1: Table
S2. They comprise publicly available databases for nondisease (for example, dbSNP, 1000 genome, UK10K, ESP6500 from NHLBI's exon sequencing project, the Wellderly project by Scripps Insititute, and ExAC data from Exome Aggregation Consortium) or diseaserelated (for example, HGMD, ClinVar, OMIM, and UK10K disease) variants. In addition, data sources that are not yet available to public are incorporated, such as genotyping data from Mount Sinai Biobank, a biobank established in 2007 in New York City with ethnically diverse participants [24], and in-house curated disease variant database VarDi [25] based on manual curation and literature mining. We also added datasets for functional consequences of the variants such as dbNSFP and pre-computed results of currently known genetic variants using tools such as SIFT [14], PolyPhen [15], ANNOVAR [7], SnpEff [26], and MutationAssessor [27].

\section{Software implementation}

ClinLabGeneticist is built on the Windows platform (Window 7 and 8). Conventional client/server architectures were utilized to support concurrent and multi-users. Specifically, the machine with Windows operating system 
Table 1 Variation annotation resources in ClinLabGeneticist

\begin{tabular}{|c|c|c|}
\hline $\begin{array}{l}\text { Variant } \\
\text { database }\end{array}$ & Description & Reference \\
\hline $\mathrm{dbSNP}$ & NCBI genetic variant database & [9] \\
\hline 1000 Genome & 1000 genome sequencing project & [35] \\
\hline ESP6500 & Exome sequencing project by NHLBI & [36] \\
\hline UK10K control & WGS cohorts of 4,000 people in UK & {$[37]$} \\
\hline $\begin{array}{l}\text { Scripps } \\
\text { Wellderly }\end{array}$ & $\begin{array}{l}\text { Sequencing of 2,000 healthy elderly } \\
\text { volunteers }\end{array}$ & [38] \\
\hline EXAC & Exome aggregation consortium & [39] \\
\hline dbNSFP & $\begin{array}{l}\text { Functional prediction and annotation of } \\
\text { non-synonymous SNVs }\end{array}$ & [40] \\
\hline HGMD & Human gene mutation database & [12] \\
\hline ClinVar & $\begin{array}{l}\text { Relationship between variants and human } \\
\text { disease phenotype }\end{array}$ & [10] \\
\hline OMIM & Online Mendelian Inheritance in Man & [11] \\
\hline UK10K disease & $\begin{array}{l}\text { WES of } 6,000 \text { patients with } \\
\text { neurodevelopment, obesity, and rare } \\
\text { diseases in UK }\end{array}$ & {$[37,41]$} \\
\hline GERA & $\begin{array}{l}\text { Genotyping data of } 78,000 \text { individuals with } \\
\text { common age-related diseases }\end{array}$ & {$[42-44]$} \\
\hline $\begin{array}{l}\text { Mount Sinai } \\
\text { Biobank }\end{array}$ & $\begin{array}{l}\text { Genotyping data from Biobank at Mount } \\
\text { Sinai }\end{array}$ & {$[24,25]$} \\
\hline VarDi & In-house disease variants database & {$[25]$} \\
\hline
\end{tabular}

for each user is the client, and the machine with the backend MySql database and performs data query, processing, and management is the server. The server is deployed in Linux. Major functions of the administrator and the reviewers such as assignment distribution, variant annotation, assignment combination, group meeting are implemented on the Windows. All of the annotated and reviewed variants by either administrator or reviewers are saved in the database on the server. The client interface is implemented by Visual Basic, HTML, and PHP.

We recommend the following hardware specifications to run the software on the client side.

- Processor - Intel ${ }^{\circ}$ core $^{\mathrm{Tm}}$ i5-3470 @ 3.20 GHz (or equivalent AMD)

- RAM - 4 GB (or higher)

- Hard drive - 120 GB 5,400 RPM hard drive

- Wireless (for laptops) - $802.11 \mathrm{~g} / \mathrm{n}$ (WPA2 support required)

- Operating system - Windows 7 or 8

Currently our backend MySql database is deployed on Mount Sinai high performance computer system which consist of 120 Dell C6145, two blade chassis nodes, 7,680 Advanced Micro Devices (AMD) $2.3 \mathrm{GHz}$ Interlagos cores (64/node) and 64 compute cores in four sockets, and 256 Gigabytes (GB)s of memory per node. A detailed instruction on software installation and setup of internal server and backend databases is available as a power point file on software's homepage [28].

\section{Patient consent and study approval}

Informed consent for clinical exome sequencing was obtained from all patients and/or their guardians. Patients assented to have their data used anonymously for research in all cases as per New York State Department of Health requirements for informed consent.

\section{Results and discussions}

\section{A comprehensive genetic variant data repository}

Our variant data repository included more than 400,000 variants at approximately 360,000 variant sites from more than 10 databases (Table 1). The total number of samples with whole genome or exome sequencing data from these databases is approximately 82,000, with an additional 90,000 genotyped individuals.

\section{Automation of clinical genetic testing process using ClinLabGeneticist}

A key feature of ClinLabGeneticist is the implementation of dashboards to automate the entire workflow. Figure 3 shows selected screenshots of the administrators' dashboard and some of the functionalities controlled by the dashboard. The dashboard (Fig. 3a) allows the administrators to upload the data (Fig. 3b), distribute the assignments with the defined timeline (Fig. 3c), highlight discordant variant evaluation results by individual reviewers (Fig. 3d), record decisions on variant interpretations and decisions on downstream validation by Sanger sequencing (Fig. 3e), and finally generate a tables of variants for the clinical report (Fig. 3f). Under hardware specification described in the software implementation section, it takes less than $10 \mathrm{~min}$ for an administrator to upload and annotate one variant file from WES. Annotation databases (Table 1) are not downloaded and stored on local servers. Instead, a link to the original database repository is provided so the administrator will always retrieve the latest annotations from each database.

Reviewers' dashboard and some of its functionalities are illustrated in Fig. 4. The dashboard (Fig. 4a) allows each reviewer to view a list of variants assigned by the administrator using the annotation databases selected by the administrator (Fig. 4b), and enables reviewers to examine relevant variant annotation data sources and references with external links in order to assess variant pathogenicity and disease association (Fig. 4c). Upon completion of evaluation, for each variant, the reviewers make a call at the gene level regarding how the phenotype of the patient relates to the disease associated with this gene (Table 2a). This is followed by a subsequent call at the variant level regarding variant pathogenic categories (Table 2b). Variant annotations from different 


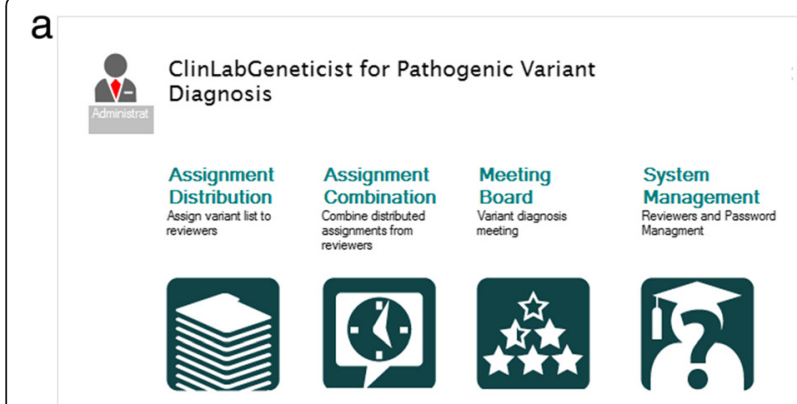

b
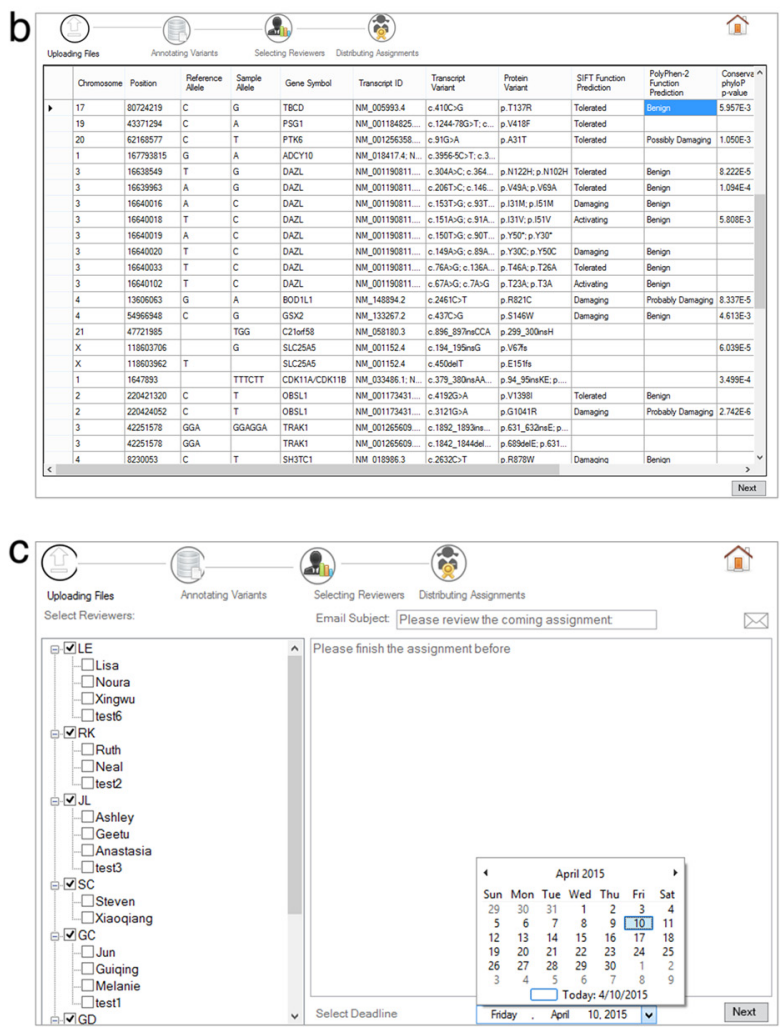

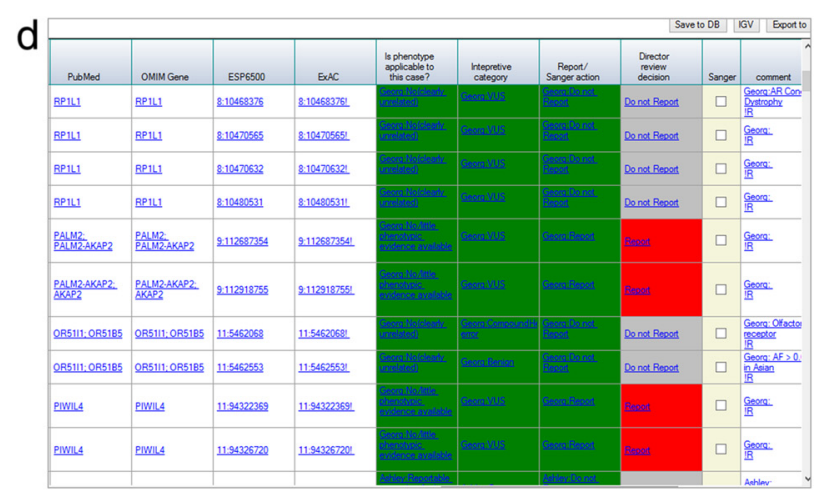

e
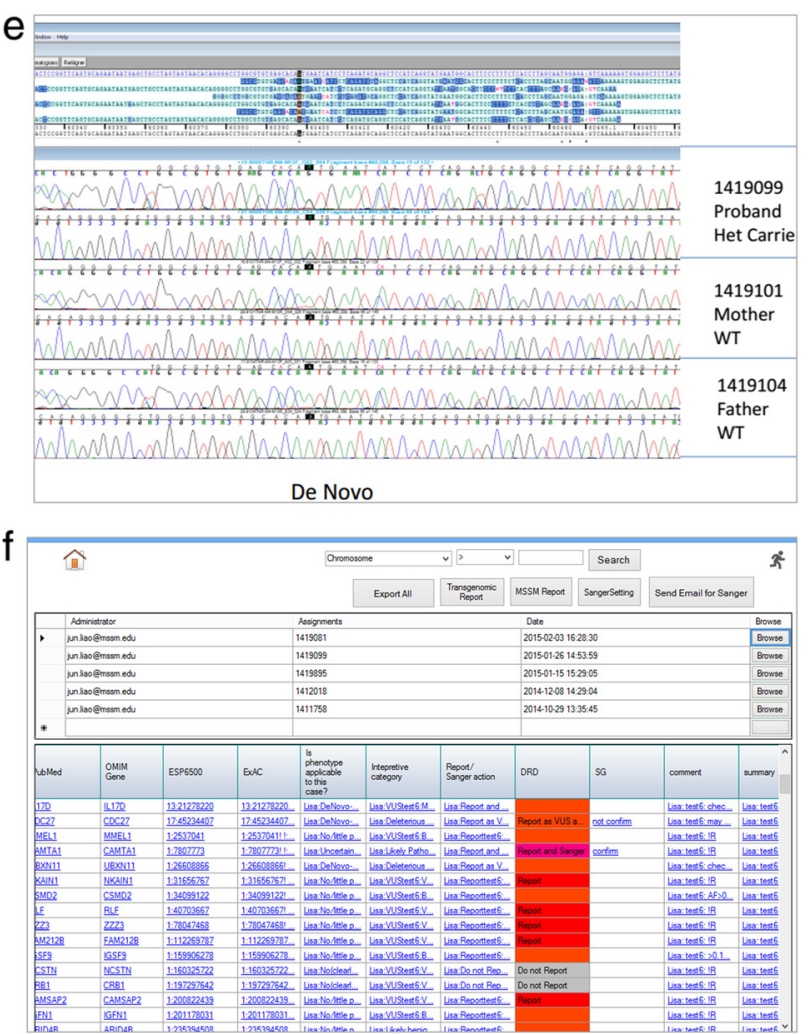

Fig. 3 Screen shots of the administrators' dashboard. (a) Dashboard, (b) functionalities controlled by the dashboard such as data upload, (c) distribute work assignments, (d) merge data table, (e) validation of variants by sanger sequencing, and (f) selection of variants to generate final reports

sources may play different roles in variant assessment depending on circumstances. For example, ClinVar, HGMD and OMIM annotations are critical to determine variant pathogenicity. Variant allele frequencies in 1000 genome and ExAC are more important parameters when variants are called benign. Based on these two calls, the ClinLabGeneticist will take the following actions for the variant based on an internally developed logic (Table 3): report and proceed to validation by Sanger sequencing, report without Sanger sequencing, or do not report. The reviewers' dashboard also allows each reviewer to browse historical assignments and review results stored in the database (Fig. 4d).
After ClinLabGeneticist was launched, we have evaluated more than 17,000 variants in 245 genes associated with 53 diseases. For most variants that lack clear evidence as pathogenic variants, it takes only 1-2 min to complete the review process using ClinLabGeneticist. For those variants with substantial annotation and literature reports, the maximal time to complete the review process is approximately $15 \mathrm{~min}$ because all relevant information is displayed by ClinLabGeneticist with external links and the IGV viewer automatically launched, allowing the reviewers to navigate the information with ease. Before ClinLabGeneticist was developed, variant Excel files were generated and distributed to each of the 

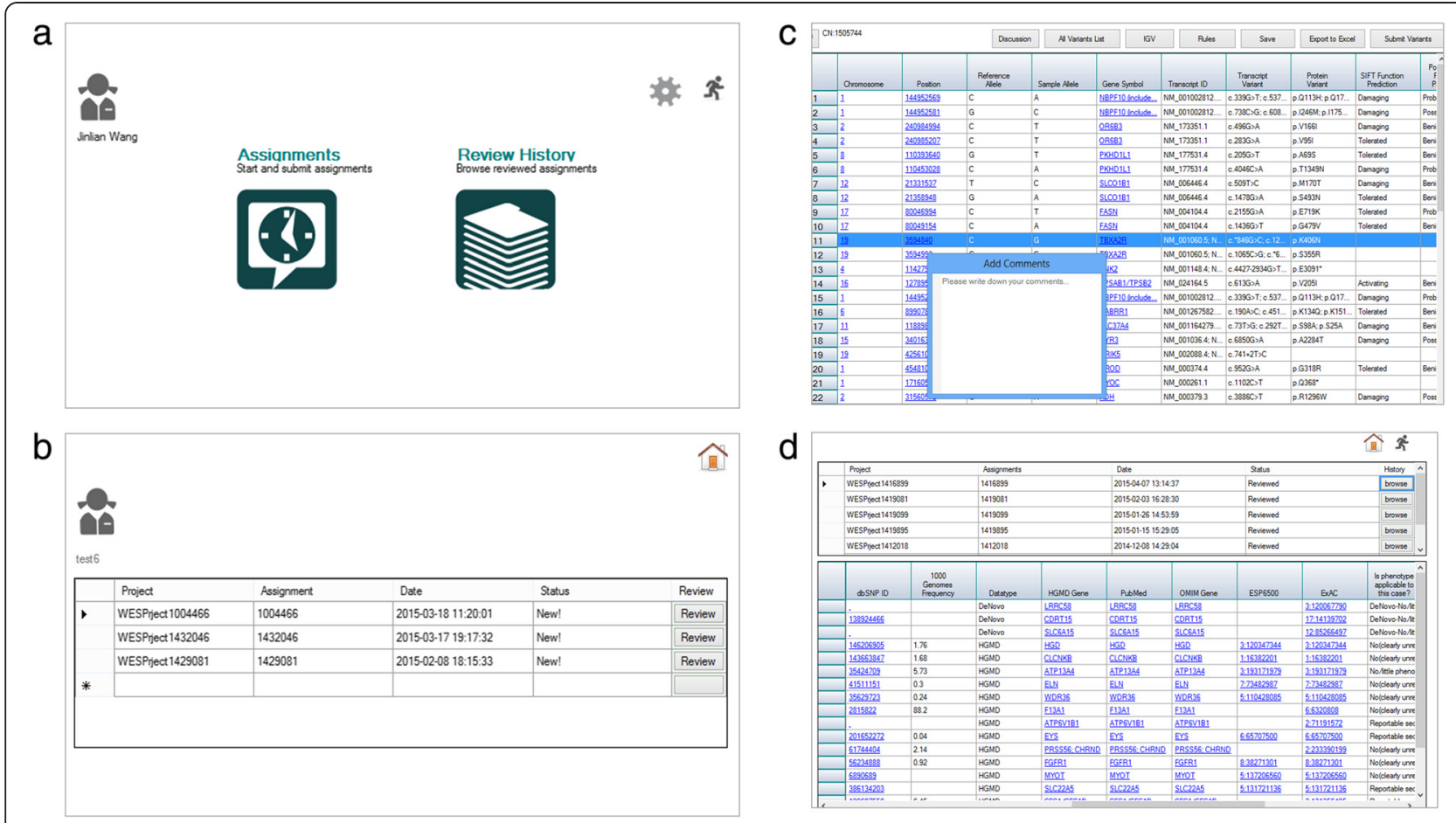

Fig. 4 Screen shots of the reviewers' dashboard. (a) Dashboard, (b) functionalities controlled by the dashboard such as display assigned variant lists, (c) review variants, and (d) access historic assignments and results

first reviewers for their variant assessment. The clinical laboratory directors or second reviewers will have to consolidate and compare first reviewers' assessment to prioritize variants for follow-up studies such as Sanger validation and categorization for final reporting. This manual workflow was transformed by the implementation of ClinLabGeneticist to become automated and therefore reduced the administrative effort by at least $50 \%$. In addition, all of the variants are annotated in ClinLabGeneticist in a fully customized manner which is essential to improve overall work efficiency and accuracy in a clinical lab. The reviewers will not need to search for annotations in different public or private databases manually. More importantly, most of the public or private variant databases are not designed for clinical use and they have to be curated and customized for clinical implementation, which can be accomplished in ClinLabGeneticist. In the following section, we present three case studies to further illustrate the utility of ClinLabGeneticist. De novo, recessive, compound heterozygous, and secondary variants in each case were analyzed. Described in Additional file 1: Table S3 are the number of variants at each step of the process, for example, concordant and discordant calls by different reviewers, decisions on variant report and Sanger sequencing validation, and variant reporting in various categories (primary, supplementary, and secondary finding). Detailed variant list for each of the three cases are provided in Additional file 1: Table S4-S6, respectively.

\section{Case study 1}

Patient 1 was diagnosed with congenital erythropoietic porphyria (CEP) at the age of 5 months by biochemical testing and the diagnosis was later confirmed by DNA analysis showing homozygosity for the UROS C73R mutation, which is known to cause a severe phenotype. The patient had a bone marrow transplantation at 2 years of age due to transfusion-dependent hemolytic anemia and severe cutaneous involvement associated with CEP. However, the patient also had several other features that were inconsistent with the diagnosis of CEP, including developmental delay, congenital glaucoma, complicated retinal and ocular problems, and facial dysmorphisms. Due to the many unexplained anomalies, the patient was evaluated by a clinical geneticist in 2012. Array CGH was normal and molecular testing for Stickler syndrome revealed a heterozygous variant of uncertain significance in the COL11A1 gene. However, these tests were performed on peripheral blood likely reflective of the bone marrow donor's results given the complete engraftment from past transplantation.

The patient was evaluated at Mount Sinai and specimens were submitted to the Mount Sinai Genetic Testing Laboratory in February 2014 for exome sequencing on fibroblasts derived from the patient's skin biopsy and blood samples from both parents. The sequence data were analyzed as a trio, and variants analysis was performed using ClinLabGeneticist software based on 
Table 2 Criteria for assessment of disease association at gene (a) and at variant (b) level

\begin{tabular}{ll}
\hline a. Is phenotype applicable to this case (at gene level) & \\
Option & Where to look \\
Yes & OMIM, HGMD, PubMed \\
Uncertain/possibly & OMIM, HGMD, PubMed \\
No (clearly unrelated) & OMIM, HGMD, PubMed \\
No/little phenotypic evidence available & OMIM, HGMD, PubMed
\end{tabular}

de novo - No/little phenotypic evidence (chose for variants from de novo filter only)

Reportable secondary finding

OMIM, HGMD, PubMed

OMIM, HGMD, PubMed

b. Interpretive category (at variant level except deleterious VUS)

$\begin{array}{ll}\text { Option } & \text { Where to look } \\ \text { Benign } & 1000 \text { Genomes, EVS, ExAC }\end{array}$

Likely benign

UCSC genome browser

Intronic-likely benign

VUS

Deleterious VUS (only chose for genes with no/little phenotype evidence)

Likely pathogenic

Pathogenic

Mapping error

CompoundHet error
UCSC genome browser, ACMG guideline

UCSC genome browser, HGMD, ClinVar

UCSC genome browser, ACMG guideline

HGMD, ClinVar, OMIM

UCSC genome browser, IGV, Ingenuity

Ingenuity
When to choose

Disease clinical features match patient's phenotype

Disease clinical features partially overlap with patient's phenotype

No overlapping phenotype, totally different disease

Phenotypic evidence was only found in few low-quality papers, or only from association studies, or only somatic mutations were reported

Same as 'No/little phenotypic evidence available', but only for de novo variants

Depends on patient's requirement, mostly for genes associated with actionable diseases. Not limit to genes in ACMG guideline. If the patient does NOT want secondary findings, do NOT choose this option

When to choose

Allele frequency $>1 \%$ for recessive or $X$-linked patterns. And for $X$-linked pattern, at least several hemizygous males should be reported in the database. Or allele frequency $>0.1 \%$ for dominant or de novo patterns

Deletion/insertion of 1-2 aa in a repeat region composed of at least 8 aa repeats

The nomenclature for all transcripts indicates that the change is intronic, but not in canonical splice sites $(-1,-2,+1$, or +2$)$, except variants reported in HGMD or ClinVar as pathogenic/likely pathogenic

Variant which does not fit other categories

Variant assumed to disrupt gene function (nonsense, frameshift, canonical splice sites, and so on), but in a gene with no/little phenotype evidence available

Has not been reported before, but is assumed to disrupt gene function (nonsense, frameshift, canonical splice sites, and so on). Or variant which meets ACMG guideline

Well-established disease-causing mutation by previous reports

Variant in segmental duplication or repeat region, and mapping quality/coverage is low. Generally you can see many variant calls in the same region. Also pay attention to complex variants such as large deletions/insertions and indels, please check IGV because nomenclature could be wrong

Only 1 non-benign variant found in a gene. Only use for variants that pass through the Compound Het filter the following inheritance patterns: de novo, autosomal recessive. ClinLabGeneticist was used in this study to evaluate seven compound heterozygous, 22 recessive, four de novo, and 15 secondary variants and generate a clinical report. From the sequencing data, a homozygous pathogenic mutation, c. $217 \mathrm{~T}>\mathrm{C}$ was identified in exon 4 of the UROS gene resulting in an amino acid change p.C73R. Mutations in UROS cause autosomal recessive congenital erythropoietic porphyria (MIM: 263700, [29]). This variant has been reported as the most frequent mutation found in CEP (CM900225 in HGMD database, RCV000003948.2 in ClinVar database, rs121908012 in dbSNP database). Sanger sequencing of DNA from the trio confirmed that the mutation was homozygous in the patient and that each of the parents was a heterozygous carrier for this variant. Therefore, the homozygous state of this variant was interpreted as a pathogenic.

Two other variants were also reported from the study. A de novo heterozygous variant of uncertain significance, c. $2855 \mathrm{G}>\mathrm{T}$ was identified in the last exon of the INPP4A gene resulting in an amino acid change p.R952L. INPP4A has not been described as a disease-related gene with substantial evidence and there is limited information in the 
Table 3 Logic for variant reporting and validation by Sanger sequencing

\begin{tabular}{|c|c|c|c|c|c|c|}
\hline \multirow[t]{2}{*}{ Interpretive category } & \multicolumn{6}{|c|}{ Phenotype applicable } \\
\hline & Yes & $\begin{array}{l}\text { No (clearly } \\
\text { unrelated) }\end{array}$ & $\begin{array}{l}\text { Reportable } \\
\text { secondary finding }\end{array}$ & Uncertain/possibly & $\begin{array}{l}\text { No/little } \\
\text { phenotypic } \\
\text { evidence } \\
\text { available }\end{array}$ & $\begin{array}{l}\text { de novo - No/little } \\
\text { phenotypic evidence (chose } \\
\text { for variants from de novo } \\
\text { filter only) }\end{array}$ \\
\hline Benign & Do not report & Do not report & Do not report & Do not report & Do not report & Do not report \\
\hline Likely benign & Report \& Sanger & Do not report & Do not report & Report \& Sanger & Report & Report \& Sanger \\
\hline Intronic-likely benign & Report \& Sanger & Do not report & Do not report & Report \& Sanger & Do not report & Do not report \\
\hline VUS & Report \& Sanger & Do not report & Do not report & Report \& Sanger & Report & Report \& Sanger \\
\hline $\begin{array}{l}\text { Deleterious VUS } \\
\text { (only chose for genes } \\
\text { with no/little } \\
\text { phenotype evidence) }\end{array}$ & $\begin{array}{l}\text { Error - please } \\
\text { change category }\end{array}$ & $\begin{array}{l}\text { Error - please } \\
\text { change category }\end{array}$ & $\begin{array}{l}\text { Error - please } \\
\text { change category }\end{array}$ & $\begin{array}{l}\text { Error - please } \\
\text { change category }\end{array}$ & $\begin{array}{l}\text { Report as } \\
\text { VUS }\end{array}$ & Report as VUS \& Sanger \\
\hline Likely pathogenic & Report \& Sanger & Need discussion & $\begin{array}{l}\text { Report as } \\
\text { secondary \& } \\
\text { Sanger }\end{array}$ & Report \& Sanger & $\begin{array}{l}\text { Error - please } \\
\text { change to } \\
\text { Deleterious } \\
\text { VUS }\end{array}$ & $\begin{array}{l}\text { Error - please change to } \\
\text { Deleterious VUS }\end{array}$ \\
\hline Pathogenic & Report \& Sanger & Need discussion & $\begin{array}{l}\text { Report as } \\
\text { secondary \& } \\
\text { Sanger }\end{array}$ & Report \& Sanger & $\begin{array}{l}\text { Error - please } \\
\text { change to } \\
\text { Deleterious } \\
\text { VUS }\end{array}$ & $\begin{array}{l}\text { Error - please change to } \\
\text { Deleterious VUS }\end{array}$ \\
\hline Mapping Error & $\begin{array}{l}\text { Investigate further } \\
\text { via Sanger }\end{array}$ & Do not report & Do not report & Need discussion & Do not report & Do not report \\
\hline CompoundHet error & $\begin{array}{l}\text { Investigate further } \\
\text { via Sanger }\end{array}$ & Do not report & Need discussion & Need discussion & Do not report & Error - not compound het \\
\hline
\end{tabular}

literature regarding its function. It has been suggested that INPP4A plays a role in brain development as targeted disruption of the Inpp $4 a$ gene in mice leads to neurodegeneration in the striatum, the input nucleus of the basal ganglia that has a central role in motor and cognitive behaviors [30]. The c. $2855 \mathrm{G}>\mathrm{T}$ variant in INPP4A is predicted to be damaging by SIFT and probably damaging by PolyPhen-2. Sanger sequencing of DNA extracted from the patient and both parents confirmed that the variant occurred de novo. A second de novo heterozygous variant of uncertain significance, c.985G $>$ A was identified in exon 11 of the $R A N B P 3$ gene resulting in an amino acid change p.E329K. RANBP3 has not been described as a diseaserelated gene with substantial evidence and there is limited information in the literature regarding its function. The variant is predicted to be damaging by SIFT and possibly damaging by PolyPhen-2. Sanger sequencing of DNA extracted from the patient and both parents confirmed that the variant occurred de novo. This variant was also interpreted to be of uncertain significance.

In addition to the above three variants, seven compound heterozygous variants were also reported in a supplementary table. For three of these seven variants, the initial review by two independent reviewers resulted in discrepant calls. In two cases, one reviewer called the variant 'VUS' while the other reviewer assigned the variant into the 'mapping error' category. In the third case, one reviewer called the variant 'likely pathogenic' and the other reviewer called the same variant 'VUS'. Upon further examination and discussion in the group meeting, it was determined that all three variants should be called 'VUS' and should be reported.

In summary, exome sequencing-based genetic testing confirmed the homozygous pathogenic mutation p.C73R despite reported complete engraftment of donor bone marrow which should have precluded a positive result. No variants were identified that explained the patient's other abnormalities though reanalysis could lead to reassignment of variant categories based on new data in the future.

\section{Case study 2}

Patient 2 had significant developmental delay and some dysmorphic features. Previous chromosome and Array $\mathrm{CGH}$ analysis had not revealed any abnormalities. DNA was also tested by a targeted gene panel for autism in the Mount Sinai Medical Genetics Testing Laboratory, but no pathogenic mutation was detected. Additional metabolic screening test results were negative.

In light of the negative metabolic and genetic testing workup, whole exome sequencing was performed on DNA extracted from the patient and the parents. The sequence data were analyzed as a trio, and variants analysis was performed using ClinLabGeneticist software based on the following inheritance patterns: de novo, autosomal recessive. A de novo variant of uncertain significance was 
identified in exon 31 of the PPFIA2 gene, NM_0012 20473.2:c.133A>G, p.Val1241Ile (hg19 Chr12:81653434). PPFIA2 has not been described as a disease-related gene with substantial evidence. This variant has not been reported in any public population variant database and is predicted to be a 'tolerated' change by SIFT in silico analysis. Sanger sequencing of DNA extracted from the patient and both parents confirmed that the variant occurred de novo. In addition, this variant was not detected in the patient's unaffected sibling.

\section{Case study 3}

Patient 3 is a 7-year-old boy with developmental delay. He had some autistic features including poor eye contact, impairment in social interaction, impairment in communication, and repetitive and stereotypic behaviors. He also had a 5-year-old brother with developmental delay. Whole exome sequencing was performed on DNA isolated from peripheral blood samples of the patient and his parents. The sequence data were analyzed as a trio, and variants analysis was performed using ClinLabGeneticist software based on the following inheritance patterns: de novo, autosomal recessive and Xlinked, and two de novo variants were identified.

The first de novo variant was identified in exon 32 of the PCNXL2 gene, NM_014801.3: c.5626C >T, p.Arg1876Cys (hg19 Chr1:233134162). PCNXL2 has not been described as a disease-related gene and there is limited information regarding its function. The variant is predicted to be damaging by SIFT and benign by PolyPhen-2. Sanger sequencing of DNA extracted from the patient, his parents and brother confirmed that the mutation occurred de novo.

The second de novo variant was identified in exon 6 of the RPS2 gene, NM_002952.3: c.623C >T, p.Pro208Leu (hg19 Chr16:2012584). RPS2 encodes a ribosomal protein that is a component of the $40 \mathrm{~S}$ subunit. It has not been described as a disease-related gene and there is limited information regarding function, although recently it has been reported that RPS2 is involved in dendritic spine maturation in rat hippocampal neurons [31]. The variant is predicted to be damaging by SIFT and benign by PolyPhen-2. Sanger sequencing of DNA extracted from the patient, his parents and brother confirmed that the mutation occurred de novo. Both de novo variant were interpreted to be of uncertain significance.

\section{Conclusions}

Advancement of next generation sequencing technologies has provided an unprecedented opportunity in medicine, and we have entered a new era of genetic and genomic testing. However, a number of barriers need to be overcome before the full potential of WES in disease diagnosis and personalized medicine can be fully realized. A constant challenge in clinical genetic testing and molecular diagnosis is to interpret the clinical significance of variants with high confidence. It has been reported that some literature-annotated pathogenic variants are not truly 'pathogenic' [32,33], and the issue is further manifested when large population exome data are examined [34]. Many variants in known disease genes that have been previously identified in specific disease cohorts occur at frequencies that are too high to support pathogenicity. Currently, there is no single comprehensive database with rigorously curated disease pathogenic variants. Therefore, it is critical to include all of the available variant annotation databases when genetic testing results are examined to assess their pathogenicity. Many commercially available variant analysis tools only include the most-commonly used population variant databases such as dbSNP and 1000 Genomes, or disease variant databases such as OMIM, HGMD, and ClinVar. ClinLabGeneticist incorporates to our knowledge, all publicly available variant databases, providing an extremely comprehensive genetic variant resource. Another issue in clinical genetic testing is the complexity of the process.

A unique feature of ClinLabGeneticist is that we implemented a logic table for variant interpretation at both gene level and variant level (Table 2). In the variant review process, it is first determined if the patient's phenotype matches clinical features of the disease associated with the gene harboring the variant. Then pathogenicity of the variant is assessed. Decisions on variant validation and reporting are made based on both gene level and variant level assessments (Table 3). In contrast, currently available tools only allow variant level evaluation and these tools are more suitable for panelbased genetic testing where only known disease genes are tested. Clearly, ClinLabGeneticist is designed to enable a more comprehensive WES-based genetic testing. Another important feature of ClinLabGeneticist is it facilitates parallel variant review by multiple reviewers, including distributing variants to different reviewers, entry of variant analysis results by the reviewers, examining results by the administrators, and decision-making on final reporting. This complex process is managed more efficiently by ClinLabGeneticist than currently available tools.

In most clinical genetic laboratories, data management and process management efficiency is suboptimal, with many tasks handled manually. ClinLabGeneticist provides a platform to streamline and automate the workflow, not only significantly improving the efficiency and scalability, but also making the entire process less error-prone. We are currently generating WES data for an average of 30 trios per month and this scale can be readily handled by ClinLabGeneticist. We do not anticipate any technical issues if the number of WES-based testing increases to 
even several hundred trios per month. The challenge though is more reviewers are needed for variant assessment as the scale of WES goes up.

We also recognize the limitation of ClinLabGeneticist. Although patient clinical information is taken into consideration during variant assessment, it has not been incorporated into ClinLabGeneticist's workflow. A new version of the software is being developed to improve on this aspect. In addition, currently ClinLabGeneticist is not amenable to analysis of disease associated copy number variation $(\mathrm{CNV})$ or chromosome structural variation (SV). Therefore, although whole genome sequencing (WGS) platform is still supported by ClinLabGeneticist, only single nucleotide variants (SNVs) and small insertions/deletions would be analyzed. We will certainly revise the workflow and the tool when more clear guidelines on $\mathrm{CNV}$ and SV assessment become available.

\section{Availability and requirements \\ Project name: ClinLabGeneticist \\ Project home page: http://rongchenlab.org/software/ clinlabgeneticist \\ Operating system(s): Windows \\ Programming language: Visual Basic, PHP, HTML \\ Other requirements: mySql \\ License: GNU, HGMD}

Any restrictions to use by non-academics: please contact the authors

\section{Additional file}

Additional file 1: Table S1. A sample variant input file for

ClinLabGeneticist. Table S2. Variant annotation databases and features. Table S3. Summary statistic of variant review process for the three case studies. Table S4. Variant list for case 1. Table S5. Variant list for case 2. Table S6. Variant list for case 3. (XLSX $42 \mathrm{~kb}$ )

\section{Competing interests}

The authors declare that they have no competing interests.

\section{Authors' contributions}

RC and LE designed and supervised the study, JW developed the software, $J L, J Z$, JW, RK, and LE performed the analysis, JW, LJ, JZ, WC, JH, MM, RR, GAD, LM, BW, SL, LE, and RC provided data and samples, SL, JW, and JL wrote the manuscript, JZ, GAD, LE, and RC revised the manuscript. All authors read and approved the final manuscript.

\section{Acknowledgments}

This work was supported in part through the computational resources and staff expertise provided by the Department of Scientific Computing at the Icahn School of Medicine at Mount Sinai.

Received: 13 April 2015 Accepted: 16 July 2015

Published online: 29 July 2015

\section{References}

1. Katsanis SH, Katsanis N. Molecular genetic testing and the future of clinical genomics. Nat Rev Genet. 2013;14:415-26.
2. Sequeiros J, Paneque M, Guimaraes B, Rantanen E, Javaher P, Nippert I, et al. The wide variation of definitions of genetic testing in international recommendations, guidelines and reports. J Community Genet. 2012;3:113-24.

3. Ingenuity Variant Analysis. Available at: http://www.ingenuity.com/products/ variant-analysis.

4. Geneticist Assistant. Available at: http://www.softgenetics.com/ GeneticistAssistant.html.

5. VarSeq. Available at: http://www.goldenhelix.com/NarSeg/index.html.

6. Mu JC, Mohiyuddin M, Li J, Bani Asadi N, Gerstein MB, Abyzov A, et al. VarSim: a high-fidelity simulation and validation framework for high-throughput genome sequencing with cancer applications. Bioinformatics. 2014;31:1469-71.

7. Wang K, Li M, Hakonarson H. ANNOVAR: functional annotation of genetic variants from high-throughput sequencing data. Nucleic Acids Res. 2010;38:e164.

8. The Exchange. Available at: https://www.nextcode.com/products-andservices/the-exchange.

9. Sherry ST, Ward MH, Kholodov M, Baker J, Phan L, Smigielski EM, et al. dbSNP: the NCBI database of genetic variation. Nucleic Acids Res. 2001;29:308-11.

10. Landrum MJ, Lee JM, Riley GR, Jang W, Rubinstein WS, Church DM, et al. ClinVar: public archive of relationships among sequence variation and human phenotype. Nucleic Acids Res. 2014;42:D980-5.

11. OMIM. Available at: http://www.omim.org.

12. Stenson PD, Mort M, Ball EV, Shaw K, Phillips A, Cooper DN. The Human Gene Mutation Database: building a comprehensive mutation repository for clinical and molecular genetics, diagnostic testing and personalized genomic medicine. Hum Genet. 2014;133:1-9.

13. Forbes $S A$, Beare $D$, Gunasekaran $P$, Leung $K$, Bindal $N$, Boutselakis $H$, et al. COSMIC: exploring the world's knowledge of somatic mutations in human cancer. Nucleic Acids Res. 2015:43:D805-11.

14. Kumar P, Henikoff S, Ng PC. Predicting the effects of coding non-synonymous variants on protein function using the SIFT algorithm. Nat Protoc. 2009:4:1073-81.

15. Adzhubei IA, Schmidt S, Peshkin L, Ramensky VE, Gerasimova A, Bork P, et al. A method and server for predicting damaging missense mutations. Nat Methods. 2010;7:248-9.

16. NCBI Resource Coordinators. Database resources of the National Center for Biotechnology Information. Nucleic Acids Res. 2015;43:D6-D17.

17. Dong C, Wei P, Jian X, Gibbs R, Boerwinkle E, Wang K, et al. Comparison and integration of deleteriousness prediction methods for nonsynonymous SNVs in whole exome sequencing studies. Hum Mol Genet. 2015;24:2125-37.

18. Aronson SJ, Clark EH, Babb LJ, Baxter S, Farwell LM, Funke BH, et al. The Genelnsight Suite: a platform to support laboratory and provider use of DNA-based genetic testing. Hum Mutat. 2011;32:532-6.

19. Linderman MD, Brandt T, Edelmann L, Jabado O, Kasai Y, Kornreich R, et al. Analytical validation of whole exome and whole genome sequencing for clinical applications. BMC Med Genet. 2014;7:20.

20. Gargis AS, Kalman L, Berry MW, Bick DP, Dimmock DP, Hambuch T, et al, Assuring the quality of next-generation sequencing in clinical laboratory practice. Nat Biotechnol. 2012;30:1033-6.

21. Green RC, Berg JS, Grody WW, Kalia SS, Korf BR, Martin CL, et al. ACMG recommendations for reporting of incidental findings in clinical exome and genome sequencing. Genet Med. 2013;15:565-74.

22. Cartagenia. Available at: http://www.cartagenia.com.

23. Clinical Sequence Analyzer. Available at: https://www.nextcode.com.

24. Streicher SA, Sanderson SC, Jabs EW, Diefenbach M, Smirnoff M, Peter I, et al. Reasons for participating and genetic information needs among racially and ethnically diverse biobank participants: a focus group study. J Community Genet. 2011;2:153-63.

25. Glicksberg BS, Li L, Cheng WY, Shameer K, Hakenberg J, Castellanos R, et al. An integrative pipeline for multi-modal discovery of disease relationships. Pac Symp Biocomput. 2015:407-418.

26. Cingolani P, Platts A, le Wang L, Coon M, Nguyen T, Wang L, et al. A program for annotating and predicting the effects of single nucleotide polymorphisms, SnpEff: SNPs in the genome of Drosophila melanogaster strain w1118; iso-2; iso-3. Fly. 2012;6:80-92.

27. Reva B, Antipin Y, Sander C. Predicting the functional impact of protein mutations: application to cancer genomics. Nucleic Acids Res. 2011;39:e118.

28. ClinLabGeneticist Installation Guideline. Available at: http://rongchenlab.org/ wp-content/uploads/2014/11/Guidline-of-ClinLabGeneticist1.pptX. 
29. OMIM Congenital Erythropoietic Porphyria. Available at: http:// www.omim.org/entry/263700

30. Sasaki J, Kofuji S, Itoh R, Momiyama T, Takayama K, Murakami H, et al. The Ptdlns(3,4)P(2) phosphatase INPP4A is a suppressor of excitotoxic neuronal death. Nature. 2010;465:497-501.

31. Miyata S, Mori Y, Tohyama M. PRMT3 is essential for dendritic spine maturation in rat hippocampal neurons. Brain Res. 2010;1352:11-20.

32. Bell CJ, Dinwiddie DL, Miller NA, Hateley SL, Ganusova EE, Mudge J, et al. Carrier testing for severe childhood recessive diseases by next-generation sequencing. Sci Transl Med. 2011;3:65ra64.

33. Wang J, Shen Y. When a "disease-causing mutation" is not a pathogenic variant. Clin Chem. 2014;60:711-3.

34. Piton A, Redin C, Mandel JL. XLID-causing mutations and associated genes challenged in light of data from large-scale human exome sequencing. Am J Hum Genet. 2013:93:368-83.

35. Abecasis GR, Auton A, Brooks LD, DePristo MA, Durbin RM, Handsaker RE, et al. An integrated map of genetic variation from 1,092 human genomes. Nature. 2012;491:56-65.

36. Fu W, O'Connor TD, Jun G, Kang HM, Abecasis G, Leal SM, et al. Analysis of 6,515 exomes reveals the recent origin of most human protein-coding variants. Nature. 2013:493:216-20.

37. Muddyman D, Smee C, Griffin H, Kaye J. Implementing a successful data-management framework: the UK10K managed access model. Genome Med. 2013;5:100.

38. Erikson GA, Deshpande N, Kesavan BG, Torkamani A. SG-ADVISER CNV: copy-number variant annotation and interpretation. Genet Med. 2014 doi: 10.1038/gim.2014.180.

39. ExAC. Available at: http://exac.broadinstitute.org.

40. Liu X, Jian X, Boerwinkle E. dbNSFP v2.0: a database of human non-synonymous SNVs and their functional predictions and annotations. Hum Mutat. 2013;34:E2393-402

41. Kaye J, Hurles M, Griffin H, Grewal J, Bobrow M, Timpson N, et al. Managing clinically significant findings in research: the UK10K example. Eur J Hum Genet. 2014;22:1100-4.

42. Enger SM, Van den Eeden SK, Sternfeld B, Loo RK, Quesenberry Jr CP, Rowel S, et al. California Men's Health Study (CMHS): a multiethnic cohort in a managed care setting. BMC Public Health. 2006:6:172.

43. Hoffmann TJ, Kvale MN, Hesselson SE, Zhan Y, Aquino C, Cao Y, et al. Next generation genome-wide association tool: design and coverage of a high-throughput European-optimized SNP array. Genomics. 2011;98:79-89.

44. Hoffmann TJ, Zhan Y, Kvale MN, Hesselson SE, Gollub J, Iribarren C, et al. Design and coverage of high throughput genotyping arrays optimized for individuals of East Asian, African American, and Latino race/ethnicity using imputation and a novel hybrid SNP selection algorithm. Genomics. 2011;98:422-30

\section{Submit your next manuscript to BioMed Central and take full advantage of:}

- Convenient online submission

- Thorough peer review

- No space constraints or color figure charges

- Immediate publication on acceptance

- Inclusion in PubMed, CAS, Scopus and Google Scholar

- Research which is freely available for redistribution 